\title{
USES OF (SE): CONTRASTIVE ANALYSIS SPANISH-ARABIC
}

\author{
Mohamed Ibrahim Mohamed Shebl ${ }^{*}$ \\ mohamed_shebl@lan.kfs.edu.eg
}

\section{Resumen:}

El análisis contrastivo constituye una herramienta que favorece el proceso enseñanza/aprendizaje de las lenguas extranjeras, puesto que proporciona las indicaciones sobre la mejor forma de enseñanza más acorde con las necesidades del estudiante. Una de las dificultades que enfrentan al alumno árabe es la de saber utilizar el elemento (se) debido a la diversidad de sus valores; ya que no existe, en su lengua materna, ningún elemento sintáctico equivalente, idéntico en su forma, que, al añadirlo al verbo enclítica o proclíticamente, presenta dicha polivalencia funcional. De este modo, este estudio de contrastes procura disponer un modelo de aproximación mental para que el profesor tenga conocimientos sobre la lengua materna del aprendiz arabo-hablante con respecto a los elementos objeto de estudio.

Palabras clave: Análisis contrastivo, sujeto agente, sujeto paciente, no-agentivo, forma básica.

\footnotetext{
Department of Spanish, Al-Alsun Faculty, Kafrelsheikh University, Kafrelsheikh, Egypt.
}

(Uses of (SE) Contrastive Analysis...) Dr. Mohamed Shebl 


\section{Introducción}

Muchos son los estudios que abordan el análisis contrastivo, su importancia y su contribución al campo de enseñanza/aprendizaje de las lenguas extranjeras, como una herramienta eficaz tanto para elaborar materiales adecuados como para formar profesores ELE conscientes de la naturaleza y la mentalidad de sus alumnos; puesto que el análisis contrastivo permite al profesor predecir los problemas que enfrentan a sus alumnos, identificar qué grado de dificultad les representa y, por lo tanto, determinar y seleccionar la mejor manera de solucionarlos.

De este modo, partiendo de nuestra plena convicción de la utilidad de dicho método pedagógico, lo hemos adoptado para llevar a cabo una contrastación entre el español y el árabe en cuanto a las cuestiones sintácticas y léxicas objeto de nuestro estudio, esto es, los valores de se en español en contraste con las unidades gramaticales equivalentes en la lengua árabe.

Sin duda alguna, uno de los errores que puede presentar el aprendiz árabe en su aprendizaje del español es el de saber utilizar el se, a causa de la diversidad de sus valores y la multiplicidad de sus usos; ya que no existe, en su lengua materna, ningún elemento sintáctico análogo, idéntico en su

(Uses of (SE) Contrastive Analysis...) Dr. Mohamed Shebl 
forma, que, al agregarlo al verbo enclítica o proclíticamente, presenta dicha polivalencia funcional: se pronombre personal, se impersonal, se pasivo, se reflexivo, se recíproco y se dativo ético.

En contraste, ante los indicados tipos y usos de la forma se, la lengua árabe dispone de muchos procedimientos y mecanismos, susceptibles de expresar los mismos hechos semántico-sintácticos, que pondremos de manifiesto con sus respectivos ejemplos y peculiaridades lingüísticas.

Así pues, la complejidad del tema seleccionado, tanto para los estudiantes árabes del español como para los que se dedican a enseñarlo; el esfuerzo de identificar y subrayar los componentes lingüísticos del árabe encargados de desempeñar los mismos valores semánticos y sintácticos; el continuo intento de llegar a una aproximación mental aceptable y útil al nivel lingüístico, constituyen el objetivo que esperamos lograr mediante el presente trabajo que investiga, entre otros logros, los puntos de contacto entre ambos idiomas -español y árabeen lo que atañe a los valores del se y sus equivalencias en el árabe.

Aún quedan dos observaciones: primero, el método adoptado para realizar este trabajo es el descriptivo; aplicado

(Uses of (SE) Contrastive Analysis...) Dr. Mohamed Shebl 
normalmente en este tipo de estudios de contrastes. Segundo, en lo que respecta a la transcripción de los fonemas árabes a lo largo del trabajo, seguiremos el modelo de la escuela de los arabistas españoles desde los años 30 , y el oficial en las revistas de Al-Ándalus (1931-1977) y Al-Qanțara (publicada desde 1980).

\section{1. (SE) PRONOMBRE PERSONAL}

1.1. En realidad, el primer uso de la forma se, en la lengua española, es el de pronombre personal. De este modo, vemos oportuno comenzar por subrayar qué constituye el se dentro de la clasificación de los pronombres personales con el fin de identificar sus peculiaridades gramaticales y destacar sus valores semánticos.

En principio, se denominan pronombres personales a unas palabras cuya función es designar a las personas gramaticales, ya sean personas, animales o cosas, haciendo referencia a las tres personas gramaticales (González Hermoso, Cuenot y Sánchez Alfaro, 2006, pp. 49-50). Tales pronombres se caracterizan por unos aspectos morfológicos y sintácticos que los distinguen de las demás categorías gramaticales del español.

(Uses of (SE) Contrastive Analysis...) Dr. Mohamed Shebl 
A este respecto, Porto Dapena afirma que: "los pronombres personales del español presentan toda una flexión basada no sólo en la distinción de personas gramaticales, sino también en el género, número, caso y reflexividad" (1986, p. 12). A su vez, Cascón Martín explica que el pronombre personal, conservando restos de la declinación latina, ofrece diferentes formas para las distintas funciones: yo, nosotros, tú, usted, vosotros, ustedes, él, ella, ellos, ellas, en función de sujeto; me, nos, te, os, en función de $\mathrm{CD}$ o $\mathrm{Cl}$; lo, la, los, las, en función de CD; le, les, se en función de $\mathrm{Cl}$ (1996, p. 18).

Pues bien, centrándonos exclusivamente en el se personal, aparte de lo que hemos expuesto arriba, pondremos de relieve lo que apunta Gili Gaya en este sentido, al decir que:

Procede del dativo latino illi, lo mismo que le, del cual se ha separado en cuanto al sonido, (...). El se personal así formado tiene el mismo valor de dativo que corresponde a le, del cual es en realidad una duplicación apta para ser usada en contacto con otras formas del mismo pronombre de tercera persona (1991, p. 235).

Llegados hasta aquí, podemos aprender que el pronombre personal se, como sustituto de le y les en función de $\mathrm{Cl}$, solo se 
utiliza cuando le sigue uno de los siguientes pronombres: lo, la, los, las en función de CD.

No obstante, Gómez Torrego indica un solo caso en que dicho pronombre puede aparecer como variante de lo en función de CD. Tal caso excepcional se da en construcciones con el verbo llamar y un predicativo: Llaman tonto a Juan. $\rightarrow$ Lo Ilaman tonto. $\rightarrow$ Se (CD) lo (predicativo) Ilaman. (2005, p. 9).

Por ende, en lo referente a su ubicación dentro del predicado, el pronombre de $\mathrm{Cl}$ se ubica antes del verbo, y cuando le acompaña un pronombre de $C D$, se coloca en primer lugar el $\mathrm{Cl}$ y le sigue el CD. Por lo demás, dichos pronombres pueden ser proclíticos, que se sitúan antes del verbo al que determinan, con todos los tiempos verbales, o enclíticos unidos al verbo, en otros casos (Diez de la Cortina Montemayor, 2012, 14. Pronombres personales III).

1.2. En la lengua árabe, existe un tipo de verbos transitivos que, por su naturaleza, tienen que ir seguidos de dos extensiones nominales, en caso acusativo, que funcionan como dos objetos: al primero de los cuales se le denomina (مفعول بٍِِ : أََّّل mafúl bihi awwal: primer objeto) y al segundo se le llama مفعول بِهِ ثانٍ: maf'úl bihi tān-in: segundo objeto) (Fayaad, 1995, pp. 45-48). En cuanto a su situación dentro de la oración, lo 
normal es que ambos objetos se pospongan al verbo; primero, el objeto que actúa, semánticamente, como $\mathrm{Cl}$, y lo sigue el otro objeto, que corresponde al CD (Al-galayeny, 2016, p.12).

De esta manera, podemos observar que el nombre, que lleva el oficio del primer objeto o $\mathrm{Cl}$, puede reemplazarse por un pronombre ${ }^{1}$ sufijado al verbo en caso acusativo. Por ejemplo, en la oración: (مَنَحْتُكَ السَّعَادَة ): (manaḥtuka as-s'àdat-a): (Te concedí la felicidad), el sufijo pronominal (كَ): (-ka) desempeña la función de primer objeto o $\mathrm{Cl}$ del verbo.

Asimismo, en el caso de que ambos objetos sean sustituidos por pronombres acusativos, continúan poseyendo su orden normal dentro de la oración. En tal caso, el pronombre

1 Nota: A modo aclaratorio y consultivo, mostraremos aquí una lista de los pronombres asilados -o autónomos- y los sufijados en caso acusativo. En primer lugar, los pronombres aislados son: (إيَّائي):

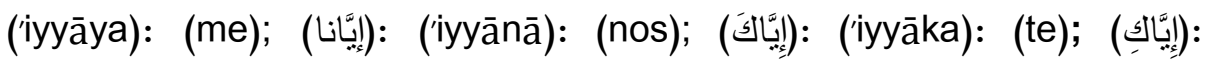

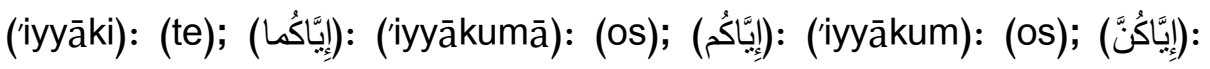

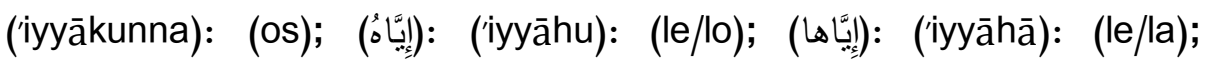

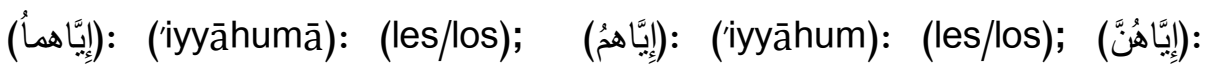
('iyyāhunna): (les/las). En segundo lugar, los pronombres sufijados son: (ه)): (hu): (lo); (ه)): (hā): (la); (كَ): (ka): (te); (ي): (ī): (me); (نا): (nā): (nos) (Awad Al-seyori (ed.), 2014/2015, pp. 12-13).

(Uses of (SE) Contrastive Analysis...) Dr. Mohamed Shebl 
personal del primer objeto tiene siempre forma sufijada y el del segundo puede tener forma sufijada o autónoma; produciendo uno de los casos siguientes: (أعُطانيكَ): ('atānīka): (Me te dio); (أعْطانيهِ): ('atānīhi): (Me lo dio); (أعُطاكَهُ): ('atākahu): (Te lo dio); (أعْطاهُ إيّالكَ): ('atāhu iyyāka): (Te lo dio); (أعْطاهُ إيّابي): ('atāhu iyyāy): (Me lo dio); (أعنطاكَ إيّائ): ('atāka iyyāy): (Te me dio) (Alsirafy, 2017, pp. 126-127).

Llegados hasta aquí, mostraremos, en seguida, unos ejemplos de todos los casos en que puede aparecer el pronombre personal se como variante de le y les, con su equivalencia en la lengua árabe:

(a) Di un libro a Juan $\rightarrow$ Le di un libro $\rightarrow$ Se lo di: (أعْطَيَتُعُ إِيَّاُه): ('a'țaytuhu 'iyyahu).

(b) Di una rosa a Juan $\rightarrow$ Le di una rosa $\rightarrow$ Se la di: (إيَّاها) أعْطَيْنُءُ ('a'taytuhu 'iyyaha).

(c) Di unos libros a Juan $\rightarrow$ Le di unos libros $\rightarrow$ Se los di: إِيَّا هُم)

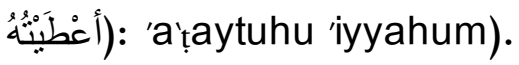

(d) Di unas rosas a Juan $\rightarrow$ Le di unas rosas $\rightarrow$ Se las di: (أعْطَيْنَهُ إِيَّاهُنَّن): ('ațaytuhu 'iyyahunna).

(e) Di un libro a María $\rightarrow$ Le di un libro $\rightarrow$ Se lo di: (أعْطَيْنُها إِيَّاهُ): ('a'țaytuha 'iyyahu).

(Uses of (SE) Contrastive Analysis...) Dr. Mohamed Shebl 
(f) Di una rosa a María $\rightarrow$ Le di una rosa $\rightarrow$ Se la di: (أعْطَيَنُها (إَّاّها ('a'țaytuha 'iyyaha).

(g) Di unos libros a María $\rightarrow$ Le di unos libros $\rightarrow$ Se los di: (أعْطَنْنُها إِيَّاهُمُ ('a'țaytuha 'iyyahum).

(h) Di unas rosas a María $\rightarrow$ Le di unas rosas $\rightarrow$ Se las di: (أعْطَيْنُها إِيَّاهُنُنَّ): ('a'taytuha 'iyyahu).

(i) Di un libro a ellos $\rightarrow$ Les di un libro $\rightarrow$ Se lo di: (أعْطَيَنُهُ إِيَّاُهُ ('a'țaytuhum 'iyyahu).

(j) Di una rosa a ellos $\rightarrow$ Les di una rosa $\rightarrow$ Se la di: (أعََْيَنُهُمْ (إَِّّاها): ('a'țaytuhum iyyaha).

(k) Di unos libros a ellos $\rightarrow$ Les di unos libros $\rightarrow$ Se los di:

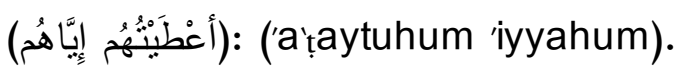

(I) Di unas rosas a ellos $\rightarrow$ Les di unas rosas $\rightarrow$ Se las di:

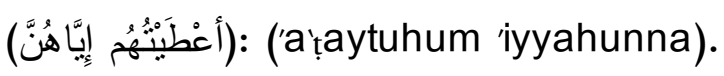

(m) Di un libro a ellas $\rightarrow$ Les di un libro $\rightarrow$ Se lo di: (أعْطَيَنُشُنَّنَ إِيَّاُه): ('a'țaytuhunna 'iyyahu).

(n) Di una rosa a ellas $\rightarrow$ Les di una rosa $\rightarrow$ Se la di: (أعْطَيَنُشُنَّنَ إيَّاِّا ها: ('a'ṭaytuhunna 'iyyahā).

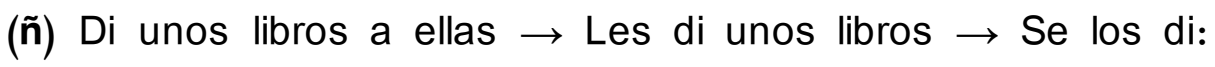

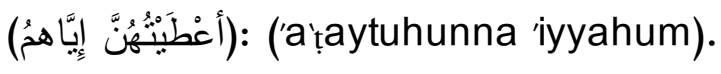

(o) Di unas rosas a ellas $\rightarrow$ Les di unas rosas $\rightarrow$ Se las di:

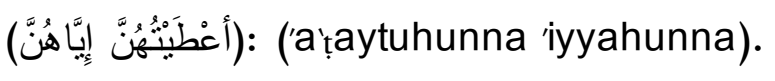

(Uses of (SE) Contrastive Analysis...) Dr. Mohamed Shebl 
(p) Di a usted el libro $\rightarrow$ Le di el libro $\rightarrow$ Se lo di: أعْطَنْكُكَه) أو إِيَّاُهُ

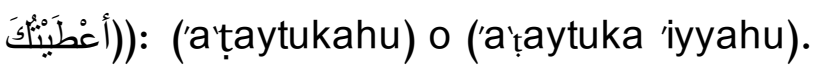

(q) Di a ustedes los libros $\rightarrow$ Les di los libros $\rightarrow$ Se los di: أعْطَيْنُمُ ('a'ṭaytukahum 'iyyahu).

(r) Da a Juan el libro $\rightarrow$ Dale el libro $\rightarrow$ Dáselo: (أعْطََ إِيَّةُه): ('a’țihi 'iyyahu).

(s) Da a Carmen la rosa $\rightarrow$ Dale la rosa $\rightarrow$ Dásela: (أعْطَها إِيَّاها): ('a'țiha 'iyyaha).

(t) Da a ellos los libros $\rightarrow$ Dales los libros $\rightarrow$ Dáselos: (إيَّاهُمْ أعْطَهِح ('a'ṭihim 'iyyahum).

(u) Da a ellas las rosas $\rightarrow$ Dales las rosas $\rightarrow$ Dáselas: (إيَّاِنُنَ) أعْطَِِنَّ): ('a'ṭhinna 'iyyahunna).

\section{2. (SE) PASIVO}

2.1. Como es bien sabido, conforme a la denominación tradicional, la gramática distingue entre la pasiva normal 0 'perifrástica' y la pasiva con se o 'pasiva refleja'. En cuanto a las estructuras del segundo tipo, Navarro Lacoba dice que: "Están formadas por el morfema se y el verbo en tercera persona. No hay complemento agente (...). El morfema se es un indicador de pasiva refleja. Se vende piso luminoso; Se venden vestidos baratos" (2014, p.2). A continuación, expondremos las propiedades formales y semánticas de las pasivas con se:

(Uses of (SE) Contrastive Analysis...) Dr. Mohamed Shebl 
En primer lugar, en la lengua española no hay morfemas específicos de voz pasiva, en consecuencia, se encargan de desempeñar ese papel: la 'pasiva perifrástica', como en el engaño fue descubierto, y el clítico se, en la 'pasiva refleja', como: se descubrió el engaño (Sánchez López (ed.), 2002. P. $50)$.

En segundo lugar, en las construcciones pasivo-reflejas la forma se se trata de un elemento auxiliar del verbo; es decir, no tiene valor pronominal y, por lo tanto, no presenta variación de persona (Cascón Martín, 1996, pp. 82-83).

Luego, en las pasivas reflejas existe un sujeto paciente, no realizador de la acción verbal, que concuerda con el verbo y, consiguientemente, dichas estructuras sirven, por lo general, para dejar indeterminado el agente del verbo (Gutiérrez Araus et. al, 2010, p. 293).

Así que, dicho sujeto de las pasivas reflejas tiene una posición postverbal; puesto que representa la información nueva que el hablante le emite al oyente; por consiguiente, no puede ser SN definido (Moreno-Fernández, Penadés-Martínez y Ureña-Tormo, 2020, p. 69).

a. Se revisará su caso.

b. Últimamente, se difunden noticias muy alarmantes.

(Uses of (SE) Contrastive Analysis...) Dr. Mohamed Shebl 
Se observa que el SN, en cursiva, funciona como sujeto paciente o sujeto gramatical no agente-realizador de acción verbal. Además, dicho sujeto debe aparecer concordado con la forma verbal.

Por último, hemos de poner de manifiesto que las pasivas reflejas y las impersonales con se comparten varias peculiaridades sintácticas y semánticas; por lo que, en muchas ocasiones, se da una confusión entre ambos tipos. Esta cuestión la trataremos en el apartado posterior.

2.2. En el idioma árabe, ante la oposición voz activa y voz pasiva, existen las estructuras de (الَََنْي للمَعْلُوم): (al-mabnī lil-malūm): (voz agentiva) y (المَنْني للمَجْهول): (al-mabn̄̄ lilmaŷhūl): (voz no-agentiva).

En lo que concierne al segundo tipo, Ibn Akil (1980), señala que cuando el verbo tiene una forma no-agentiva, se produce un proceso de transformación mediante el cual se sustituye el sujeto agente por el objeto del verbo. Así pues, en este caso, con la eliminación del sujeto, el objeto del verbo -que iba en caso acusativo- pasa a funcionar como sujeto paciente en caso nominativo, no susceptible de anteponerse al verbo; como si fuera un sujeto agente desde el punto de vista sintáctico. Sin embargo, adoptando un criterio semántico, dicho

(Uses of (SE) Contrastive Analysis...) Dr. Mohamed Shebl 
nombre sigue funcionando como recibidor de la acción verbal realizada por un sujeto desconocido o no mencionado en la frase. A este respecto, conviene observar que la voz noagentiva del verbo, en árabe, implica la utilización de la 2a persona singular, 3. persona plural, y la agregación del prefijo con vocal /u/. (p. 111-114). Para clarificar lo indicado arriba, daremos los siguientes ejemplos:

a. (كَنَبَ الطَّالِبُ الدَّرسَ): (Katab-a at-țālib-u ad-dars-a): (Trad. Lit.: Escribió el alumno la lección).

b. (كُنَبَ الدَّرسُ): (Kutiba ad-dars-u): (Se escribió la lección).

La oración (a), se trata de una oración verbal encabezada por un verbo en perfectivo, seguido del sujeto y, luego, el objeto que es un $\mathrm{SN}$ en caso acusativo. Al transformar dicha oración a la voz no-agentiva, se da el caso (b); donde pasó el objeto a desempeñar la función de sujeto paciente del verbo, tras la eliminación de sujeto oracional, por medio del uso del prefijo con vocal $/ \mathrm{u} /$.

c. (يَتَرْبُ الرَّجُلُ الماءً): (Yašrab-u ar-raŷul-u al-mā'-a): (Trad. Lit.: Bebe el hombre el agua).

d. (يُشَرْبُُ الماءُ): (Yušrab-u al-mā'-u): (Se bebe el agua).

De igual modo, los casos (c) y (d) constituyen ejemplos de la voz agentiva y la no-agentiva respectivamente. Puesto que,

(Uses of (SE) Contrastive Analysis...) Dr. Mohamed Shebl 
luego de la elipsis del sujeto, el SN funcionando como complemento en caso acusativo se convierte en sujeto paciente en caso nominativo recibiendo, semánticamente, la acción expresada por el verbo que sufre, por lo tanto, unos cambios formales necesarios para que se logre la forma no-agentiva.

\section{3. (SE) IMPERSONAL}

3.1. Las construcciones consideradas como impersonales son muy variadas en la lengua española. Así, para precisar el significado general de la impersonalidad, Sánchez López (ed.) dice que: "En general, se suele considerar impersonal toda oración que carece necesariamente de sujeto explícito" (2002, pp. 18-19).

En efecto, entre los recursos más importantes que expresan la impersonalidad, en español, se destaca el uso de las construcciones con se, donde se centrará nuestro estudio en este apartado. A continuación, subrayaremos sus peculiaridades sintácticas y semánticas:

El primer rasgo, como se ha señalado arriba, es que en ningún caso hay posibilidad de determinar el sujeto en este tipo de estructuras.

El segundo rasgo que salta a la vista, consiste en que el se impersonal, igual que el se de las secuencias pasivo-reflejas,

(Uses of (SE) Contrastive Analysis...) Dr. Mohamed Shebl 
es un elemento auxiliar del verbo que no tiene valor pronominal $y$, en consecuencia, no ofrece variabilidad de persona (Cascón Martín, 1996, pp. 82-83).

El tercero, reside en que la interpretación inespecífica de las impersonales con se se debe a la ausencia del sujeto. Así pues, en virtud de Sánchez López (ed.) (2002), para interpretar este tipo de secuencias, tenemos dos posibilidades: una interpretación genérica y una interpretación existencial; según las cuales, el sujeto puede interpretarse de forma similar a 'todo el mundo' o 'cualquiera', en la primera, y a 'alguien', en la segunda, en función de las características léxico-semánticas del predicado (pp. 27-28). Para ejemplificar lo expuesto en este párrafo, se puede considerar los casos que siguen:

a. Se ayuda mucho a los inmigrantes.

b. Se vive muy bien en esa ciudad.

c. Aquí se fuma demasiado.

Puesto que, de acuerdo con lo mencionado, podemos parafrasear los casos (a), (b) y (c) de la siguiente manera: Todo el mundo ayuda a los inmigrantes; Cualquiera vive bien en esa ciudad; Alguien fuma demasiado.

Por otro lado, cabe notar que no se puede formular expresiones impersonales con se, cuando los verbos son

(Uses of (SE) Contrastive Analysis...) Dr. Mohamed Shebl 
pronominales puros (quejarse, atreverse, jactarse, etc.). En este caso, se suele recurrir a otro procedimiento (A. de Molina Redondo, 1990, p.16).

Así que, en muchas ocasiones, cuando el verbo es transitivo, se produce una confusión entre las impersonales con se y las pasivas con se, ya que ambos tipos comparten varias propiedades.

En este sentido, A. de Molina Redondo (1990) explica que el criterio semántico no es válido a la hora de establecer diferencias entre una y otra clase de se; impersonal y pasiva. Por consiguiente, la oración impersonal (Se vende pan) puede ser entendida con sentido pasivo, y, de forma igual, una oración pasiva como (Se recibió el giro ayer) puede recibir una interpretación impersonal (p. 20).

En efecto, para evitar este tipo de confusión, por nuestra parte, preferimos seguir los pasos de Cascón Martín, al decir que: "Habitualmente los verbos intransitivos dan lugar a construcciones impersonales, mientras que los transitivos suelen producir pasivas (...). Sin embargo, cuando el verbo transitivo tiene un CD precedido de a, solo hay impersonalidad" (1996, pp. 82-83). Dando para el último caso el siguiente ejemplo: Se convocó a los opositores.

(Uses of (SE) Contrastive Analysis...) Dr. Mohamed Shebl 
3.2. En relación con la lengua árabe, se ha aludido a la oposición entre la voz agentiva y la voz no-agentiva en el verbo árabe; sirviendo esta última para predicar sobre acciones cuyo sujeto está elidido, poseyendo, en consecuencia, el mismo valor de las pasivas reflejas en español.

Partiendo de la coincidencia sintáctica y semántica entre las pasivas con se y las impersonales con se, podemos afirmar que la voz no-agentiva puede ser una oportuna selección a la hora de querer representar secuencias gramaticales, en árabe, equivalentes a las oraciones impersonales. A este respecto, nos parece conveniente destacar lo que dice Corriente (2006) al respecto:

La voz no-agentiva sirve para expresar juicios impersonales, en los que el sujeto es indiferente, vgr., يُعَالُ «se dice». En árabe, esto se puede hacer también mediante la 2.a persona singular, 3a persona plural,

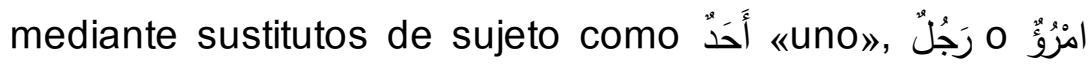
«un hombre» (p. 163).

De este modo, el primer hecho que conviene mencionar, aquí, es que el gramático, en su referencia al empleo de la voz no-agentiva, en el árabe, como unidades análogas a las impersonales con se, indica que dicho valor semántico puede

(Uses of (SE) Contrastive Analysis...) Dr. Mohamed Shebl 
lograrse también por medio de uno de los sustitutos apropiados de los que dispone el árabe, esto es, la utilización de nombres

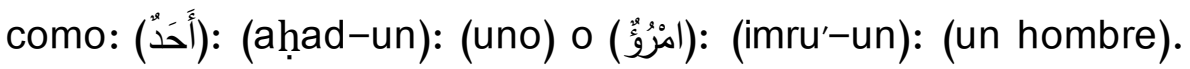

Así pues, desde nuestro punto de vista observamos que tal comportamiento lingüístico coincide con la interpretación inespecífica de las impersonales con se, señalada anteriormente, según la cual el sujeto puede interpretarse de forma similar a 'todo el mundo', 'cualquiera' o 'alguien'.

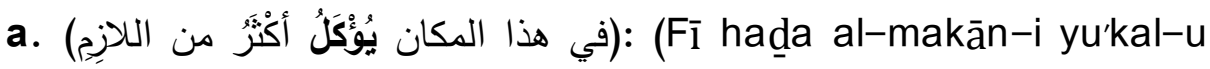
akțar-u mina al-lāzim-i): (En este lugar se come demasiado).

b. (يَعيشُ المَزْوُُ أفضْنَ في الريف): (Ya`šš-u al-mar'-u afḍal fī ar-rīf-i): (Se vive mejor en el campo).

c. (مع الأسف لا يَحْنَرِم أحَدُ الكِبارَ): (Ma'a al-'asaf lā yaḥtarim aḥad-un al-kibār-a): (Lamentablemente no se respeta a los mayores).

\section{4. (SE) REFLEXIVO}

4.1. En lo que atañe al significado de reflexividad en la lengua española, los gramáticos opinan que tal concepto lingüístico radica en que el sujeto y el objeto (directo o indirecto) tienen el mismo referente, dicho de otro modo, el sujeto realiza una acción que recae sobre él mismo (Sarmiento y Sánchez, 1989, p. 97). Esto quiere decir que la reflexividad, como fenómeno lingüístico presente en español, se produce cuando el

(Uses of (SE) Contrastive Analysis...) Dr. Mohamed Shebl 
sujeto de una oración y el pronombre reflexivo se, que desempeña, en este caso, la función de $(\mathrm{CD}) \circ(\mathrm{Cl})$, coinciden en un mismo ser.

En efecto, la utilización de los pronombres reflexivos, de conformidad con Cascón Martín (1996), se trata de un mecanismo creado por el idioma con el fin de evitar las redundancias no necesarias; por lo cual, en lugar de decir Manuel lava a Manuel, decimos: Manuel se lava (p. 80). Asimismo, el valor de reflexividad se realiza cuando la acción del verbo se refleja en una parte del cuerpo o la ropa del sujeto. Ej. Me he cortado un dedo, Me he roto la falda (Aragonés y Palencia, 2009, p. 66).

Por otro lado, hay que advertir que el se reflexivo tratado aquí, a diferencia del se personal, es susceptible de alternarse con los pronombres de primera y segunda persona me, te, nos y os.

a. Los animales se asustaron.

b. ¿A qué hora os habéis despertado hoy?

A modo de introducción, para tratar las oraciones analizadas como reflexivas y, por tanto, identificar las unidades sintácticas que funcionan de forma similar en la lengua árabe, sugerimos exponer algunos criterios, establecidos por los

(Uses of (SE) Contrastive Analysis...) Dr. Mohamed Shebl 
gramáticos, en español, según los cuales dichas estructuras con se se dividen en subclases:

En primer lugar, en virtud del oficio que lleva el pronombre reflexivo como $\mathrm{CD}$ o $\mathrm{Cl}$, tales oraciones se dividen en reflexivas directas e indirectas respectivamente (Gili Gaya,1991, pp. 7374). Para ilustrar su explicación, da los siguientes ejemplos: Luis se ha peinado (CD), es reflexiva directa; Luis se ha puesto un sombrero nuevo $(\mathrm{Cl})$.

En segundo lugar, partiendo de un punto de vista semántico, tales estructuras se clasifican según que los verbos pronominales sean de cambio de estado (secarse, mojarse, hundirse, ...), de reacción emocional (entristecerse, contentarse, equivocarse, ...) o reflexivos propios (acatarrarse, afiebrarse, arrepentirse, jactarse, ...) (Sánchez López (ed.), 2002, pp. 72106).

Además, adoptando un criterio formal y/o semántico, Aragonés y Palencia (2009) presentan una descripción muy sucinta de los verbos con $(m e, t e, s e, \ldots)$ clasificándolos del siguiente modo: verbos que solo poseen la forma con se (arrepentirse, quejarse, atreverse, ...); verbos que pueden aparecer sin el pronombre reflexivo para indicar que la acción del verbo, realizada por el sujeto, recae sobre un objeto que no

(Uses of (SE) Contrastive Analysis...) Dr. Mohamed Shebl 
coincide con el propio sujeto (acostarse y acostar, aburrirse y aburrir, ...); verbos que tienen significados distintos con o sin el pronombre se (dormir y dormirse; dejar y dejarse; llevar y llevarse, etc.) (pp. 98-100).

c. Ana se queja de los profesores; No me arrepiento de nada.

d. Acuesto a los niños; Me acuesto temprano.

e. ¿Cuántas horas duerme Javi al día?; Javi siempre se duerme en la ópera.

Pues, cada ejemplo representa uno de los tipos manifestados arriba. A tal efecto, observemos que el verbo, en el tercer caso, cuando se utiliza como verbo pronominal, su significado cambia de descansar a aburrirse.

Por su parte, Cascón Martín (1996) añade a las clases de verbos, antes citadas, un tipo de verbos que aceptan las dos construcciones sin cambio sustancial en el significado: (confesar y condesarse; vengar y vengarse; admirar y admirarse, ...) ( $p$. 82).

f. El partido vengará vuestra muerte.

g. Venus se venga de Hipólito.

Habida cuenta de que la diferencia principal entre ambas frases consiste en el tipo de complemento que se rige en cada caso.

(Uses of (SE) Contrastive Analysis...) Dr. Mohamed Shebl 
4.2. En principio, la lengua árabe carece de la reflexividad, tal y como está empleada en la lengua española; puesto que no hay serie de pronombres que, al añadirlos a la forma básica -o tema- del verbo, este adquiere un valor reflexivo como sucede en el castellano.

Sin embargo, en el árabe existen otras maneras según las cuales se puede alcanzar el significado oracional adquirido mediante el pronombre reflexivo se. A este respecto, desde nuestro punto de vista, para realizar una contrastación significativa, entre el español y el árabe, en relación con las estructuras en cuestión, nos hemos de basar en los planteamientos, antes expuestos, en torno a la caracterización de los verbos reflexivos en español. De esta forma, podemos hallar lo siguiente:

4.2.1. Primero, a base de la subdivisión de las reflexivas en directas e indirectas, en las oraciones del primer tipo donde recae la acción verbal sobre el propio sujeto, el árabe presenta unos mecanismos para describir tal caso:

En un principio, la morfología árabe es la disciplina que se ocupa del estudio de las formas básicas -o temas- de las palabras y de las normas de combinación de morfemas que forman parte de las mismas. En este sentido, los gramáticos

(Uses of (SE) Contrastive Analysis...) Dr. Mohamed Shebl 
establecieron un modelo o paradigma morfológico denominado (الميزان الْصَّرْفِيّة): (al-mīzān aṣ-ṣarfiyy), según el cual se puede precisar e identificar la forma básica de la palabra por medio de subdividirla en un morfema radical -o lexema- y un o unos morfemas derivacionales ligados considerados como morfemas no-radicales.

Por lo demás, como la mayoría de las palabras árabes se componen de tres letras consonantes radicales, se eligieron las

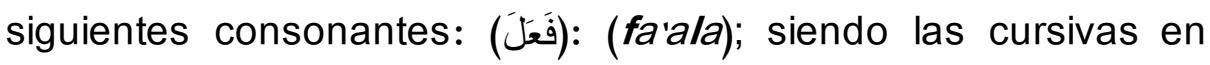
negrita las que simbolizan dichas consonantes. Así que, cuando el verbo está compuesto de cuatro letras consonantes, se duplica la última letra del modelo antes mencionado, equivaliéndose a la forma: (فَعْلَكَ): (fallala). Por ejemplo, la palabra (كَ): (kataba): (escribió), tiene la forma (fa-'a-la) y la palabra (زَ)َزَلَ): (zalzala): (sacudió), posee la fórmula: (fa-'-lala). De esta manera, a partir del indicado paradigma morfológico, existe un grupo de letras, o mejor dicho morfemas no-radicales, que pueden aparecer prefijadas, infijadas o sufijadas al lexema de la palabra. Tales morfemas, consideradas como no-radicales, se agrupan en la palabra árabe:

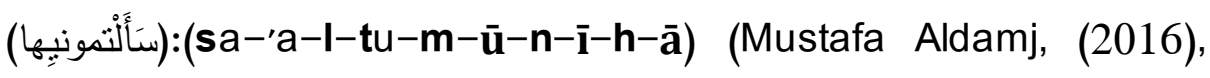
pp. 17-19).

(Uses of (SE) Contrastive Analysis...) Dr. Mohamed Shebl 
Así pues, desde nuestro punto de vista, el empleo de dichas letras o morfemas no-radicales afijados a la forma básica del verbo árabe, es el que conlleva el sentido de la reflexividad del verbo castellano. Sirven de ejemplos los siguientes:

a. (غَسَلَ): (gasala): (lavó).

b. (اغْنَسَلَ): (igtasala): (se lavó).

Se nota que el verbo (غَسَلَ), en (a), tiene la forma básica (fa-'a-la) y funciona como verbo transitivo. Mientras que el verbo (اغْنَسَلَ), cuyo efecto se refleja sobre el propio sujeto, se compone de la misma forma básica además de unos morfemas no-radicales.

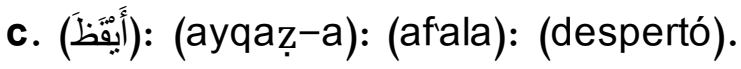

d. (اسْنَنَفَمًَ (istayqaza): (istafala): (se despertó).

Como se ve, la diferencia entre ambos ejemplos consiste en que el verbo, en (c), requiere un objeto, y en (d) funciona como verbo reflexivo gracias a la presencia de los morfemas derivacionales ligados al lexema o morfema base de la palabra.

4.2.2. Respecto a las oraciones del segundo tipo, donde recae la acción verbal sobre una parte del cuerpo o la ropa del sujeto, la lengua árabe posee otro procedimiento para expresar dicho valor.

(Uses of (SE) Contrastive Analysis...) Dr. Mohamed Shebl 
Puesto que, en árabe, los pronombres personales ${ }^{2}$ sufijados funcionan como posesivos cuando se sufijan a un nombre. Por ejemplo, (صَديقي): (șadīq-ī): (mi amigo), donde funciona $/-\overline{\mathrm{i}} /$ como pronombre personal posesivo de 1. apersona singular. En tal sentido, debemos advertir que, a diferencia de los posesivos en español, dichos pronombres que funcionan como posesivos solo señalan el género y número del poseedor (Awad Al-seyori, Abdul-Hamid (ed.), 2015, p. 13-14).

a. (Me lavo las manos): (أغَسِلُ يَديَّ): (Agsil-u yadayy-a).

b. (Te mojaste la cara): (بَلَتْت وَجْهُكَ (Balalta waŷhak-a):

c. (Se quitó la camisa): (نَزَعَ قَمَيصَنَ): (Naza'-a qamīṣah-u).

Por consiguiente, podemos decir que, en árabe, la existencia de tales posesivos sufijados al nombre, cuya función

2 Nota: Los pronombres personales sufijados en función de posesivos son los siguientes: 1. a pers. del singular, (ي) (-ī); 2a pers. del singular: (كَ): (-ka) para el masculino, y (كَ) : (-kī) para el femenino; 2. pers. del dual: (sَ): (-kumā); 2a pers. del plural: (sُ): (-kum) para el masculino, y (sُنَّ): (-kunna) para el femenino; 3a pers. del singular: (\$ُ): (-hū) para el masculino, y (هَ): (-hā) para el femenino; 3. pers. del dual: (هُهَ): (-humā); 3apers. del plural: (هُمَا): (-hum) para el masculino, y (هُ): (-hunna) para el femenino; 1 a pers. del plural: (ن): (-nā) (Awad Al-seyori, Abdul-Hamid (ed.), 2015, p. 13-14).

(Uses of (SE) Contrastive Analysis...) Dr. Mohamed Shebl 
es $C D$, es la que conlleva el valor semántico adquirido, en español, en las reflexivas indirectas.

\section{5. (SE) RECÍPROCO}

5.1. En términos generales, el pronombre recíproco se se usa de la misma forma que el se reflexivo, con la diferencia de que, en este caso, el sujeto es múltiple y, en consecuencia, se considera que cada individuo del sujeto ejecuta la acción verbal hacia el otro o los otros. Esto viene de acuerdo con Cascón Martín cuando señala que: "La reciprocidad implica una acción mutua de dos o más sujetos, cada uno de los cuales se convierte en objeto de lo que hace el otro" (1996, p. 81). El mismo gramático, para ilustrar su argumento da los siguientes ejemplos: Manuel y Manuela se quieren y Manuel y Manuela se dan besos amorosos; donde funciona el pronombre se como $\mathrm{CD}$, en el primero, y $\mathrm{Cl}$, en el segundo.

Dicho de otro modo, para que tenga lugar un valor recíproco, el se recíproco requiere un sujeto plural; por lo cual, el verbo siempre está conjugado en plural al igual que el pronombre átono que lo acompaña: nos, os y se. Tal reciprocidad se puede comprobar con las expresiones mutuamente, unos a otros, entre sí (Sarmiento y Sánchez, 1989, p. 98).

(Uses of (SE) Contrastive Analysis...) Dr. Mohamed Shebl 
a. Mi novia y yo nos celamos bastante.

b. Martina y Clara se desafían todo el tiempo.

c. Se golpearon sin querer mientras caminaban en direcciones opuestas.

5.2. Se ha adelantado que, conforme al paradigma de la morfología árabe, las palabras se forman a través de la intersección de un morfema radical y un o unos morfemas noradicales. Como resultado de dicha intersección, se han formado ciertas secuencias fijas, denominadas formas. Cada una de dichas formas posee su propio significado, de modo que, cuando un verbo adopta una de ellas, adquiera un valor semántico particular.

En efecto, para denotar la noción de reciprocidad, tal y como está existente en el español, la lengua árabe presenta varias fórmulas dotadas, entre otros usos, de expresar dicho valor semántico. Las más frecuentes son:

5.2.1. (تَفَاعَلَ): (tafā'ala). Esta fórmula sirve para indicar que cada individuo, de los que forman el sujeto, realiza la acción del verbo y la recibe mutuamente. Además, cuando aparece el verbo adoptando tal forma, puede ser transitivo o intransitivo (Gawad Altereehy, 2014, p. 1). Con el fin de clarificar lo citado, el autor da los ejemplos que siguen:

(Uses of (SE) Contrastive Analysis...) Dr. Mohamed Shebl 


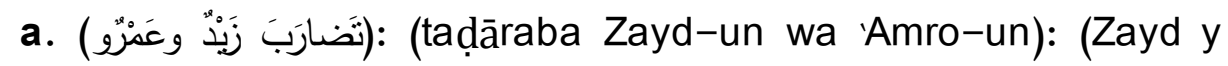
Amro se pegaron).

b. (تََازَعَ زَيْدُ وعَمْرُو الأمْرَ): (tanāza'a Zayd-un wa 'Amro-un al-'amra): (Zayd y Amro se discutieron el asunto).

5.2.2. (إْتَعَلَ): (ifta'ala). Esta fórmula, también, puede expresar la reciprocidad (Mostafa Aldamg, K., (2016), p. 46). Por ejemplo:

c. (اختَََنَ الرَّجُلان): (Jtașama ar-raŷulān): (Los dos hombres se regañaron).

5.2.3. (تَفاعَلَ): (tafā'ala). Uno de los usos de esta fórmula es su capacidad de denotar reciprocidad (Mostafa Aldamg, K., (2016), p. 49). Dando el ejemplo siguiente:

d. (تَمَاهَا وتَعَانقام): (tafāhmā wa ta'ānaqā): (se comprendieron y se abrazaron).

\section{6. (SE) DATIVO ÉTICO}

6.1. En ocasiones, el pronombre reflexivo funciona, desde el punto de vista semántico, como intensificador del proceso verbal. En cuanto a este tipo de construcciones, Gili Gaya dice que "la reflexión del acto puede atenuarse de modo que los pronombres no sean ya complemento directo o indirecto, sino que indiquen vagamente una participación o interés en la acción

(Uses of (SE) Contrastive Analysis...) Dr. Mohamed Shebl 
producida"(1991, p. 74). Gili Gaya considera que este rasgo lingüístico involucra desde el llamado dativo ético o de interés (Ella se tomó el café) hasta las llamadas expresiones pseudorreflejas (Te estás en casa; Me salí del despacho).

A su vez, A. de Molina Redondo (1990), con el fin de estudiar dicho comportamiento, presenta una lisa de verbos que, según él, no es completa, pero es bastante amplia: almorzar, cenar, comer, desayunar, engullir, merendar, tomar, beber, tragar, fumar, aprender, conocer, creer, imaginar, saber, suponer, temery otros (p. 63).

En este caso, su función de $\mathrm{Cl}$ en estructuras transitivas o intransitivas, es marginal, por lo que se puede suprimir sin que cambie el significado en esencia. Ej. Ya me (Cl) he estudiado dos temas (yo); El niño se (Cl) comió el bollo. (Cascón Martín, 1996, p. 81). Así pues, se nota que el pronombre reflexivo, denominado dativo ético, puede añadir matices significativos, no siempre precisos ni claros.

Por su parte, Aragonés y Palencia opinan que este uso intensificador del pronombre se resalta cuando se habla de cantidades. Ej. Enrique come muchos bocadillos $\rightarrow$ Enrique se come dos bocadillos (2009, p. 98).

(Uses of (SE) Contrastive Analysis...) Dr. Mohamed Shebl 
Pues bien, a la luz de lo que expone A. de Molina Redondo (1990) acerca de este tipo de secuencias, se puede decir que, la inserción de un pronombre que representa al sujeto, en este caso, sirve para enfatizar la relación entre el sujeto y un objeto determinado. Además, los efectos producidos, según él, es algo que tiene su lugar en el estudio del léxico mejor que en el de la sintaxis (p. 69).

a. Ayer me comí una paella y un filete asado.

b. ¿Te has aprendido la lección?

c. Se ha ganado un premio.

6.2. De hecho, no podemos decir que, en la lengua árabe, haya una unidad sintáctica específica que tenga la función del dativo ético se. Sin embargo, a partir del valor léxico y semántico de dicho pronombre y la intensidad que daría al sujeto de la oración, nos parece adecuado acudir al estilo de afirmación en la lengua árabe:

La verdad es que la afirmación es de dos tipos, léxica y semántica. En cuanto al primer tipo, se da mediante la repetición del vocablo que se desea enfatizar en la oración. Mientras que la afirmación semántica o ética se produce por medio del uso de unos términos particulares a los cuales se sufijan pronombres personales que concuerdan con el sujeto

(Uses of (SE) Contrastive Analysis...) Dr. Mohamed Shebl 
objeto de enfatización (Osama Kamel, 2004, pp. 122-126). Como explicación y como ejemplo de lo dicho, pueden servir los siguientes:

a. (الحُرِيَّة الحُرَيَّة أفْضَلُ سَبِيل): (al-hurriyyah Afḍal-u sabīl-in): (Trad. Lit. La libertad la libertad el mejor camino).

b. (جاءَ الأميرُ نَفَفْنُةُ): (ŷā'-a al-'amīr-u nafsuh-u): (Trad. Lit. Vino el príncipe mismo).

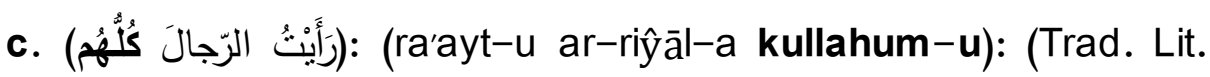
Vi a los hombres a todos).

d. (حَضَرَ الطُّلابُ عامَتَُْهم): (ḥaḍar-a aț-țullab-u ammatuhum): (Trad. Lit. Asistieron los alumnos su totalidad).

Como se puede notar, el primer caso es un ejemplo de la afirmación vocal; donde sirve la repetición del nombre para enfatizar su presencia en la oración. Por otra parte, los casos (b), (c) y (d) constituyen ejemplos de la afirmación semántica; puesto que la aparición de tales vocablos, en negrita, cuya presencia es prescindible, refuerza el significado general de la secuencia.

\section{Conclusiones}

Pese a la divergencia entre los sistemas lingüísticos español y árabe, podemos afirmar que el contraste entre ambas lenguas puede tener su potencial para disminuir algunas de las

(Uses of (SE) Contrastive Analysis...) Dr. Mohamed Shebl 
dificultades surgidas en el proceso enseñanza/aprendizaje del español. Así, a partir del análisis contrastivo que hemos llevado a cabo, hemos podido concluir lo siguiente:

a. El primer uso del se, en español, es el de pronombre personal sustituto de le y les en función de $\mathrm{Cl}$, cuando lo sigue un pronombre de CD. A este respecto, ambos pronombres se sitúan antes del verbo, se coloca primero el se $y$, posteriormente, el pronombre cuya función es CD. Aun así, en la lengua árabe, cuando se reemplazan los dos objetos del verbo, cuya función semántica es la de $\mathrm{Cl}$ y $\mathrm{CD}$ respectivamente, por pronombres en caso acusativo, estos se posponen al verbo: primero el del $\mathrm{Cl}$ siempre en forma sufijada, y luego el del CD en forma sufijada o autónoma.

b. En la lengua española, en las pasivas reflejas, la voz pasiva se logra mediante el clítico se y un sujeto paciente, pospuesto al verbo, no realizador de la acción verbal, que concuerda con el verbo. Dichas estructuras sirven, por lo general, para dejar indeterminado el agente del verbo. De un modo análogo, la lengua árabe dispone de la voz no-agentiva: estructuras formadas por un verbo en 2a persona singular o 3 a persona plural + un prefijo con vocal /u/y un sujeto paciente, no susceptible de anteponerse al verbo, no realizador de la acción

(Uses of (SE) Contrastive Analysis...) Dr. Mohamed Shebl 
verbal. También, tales secuencias se utilizan cuando el sujeto es eliminado.

c. A partir de la convergencia sintáctica y semántica entre las pasivas con se y las impersonales con se, se puede considerar que la voz no-agentiva, del idioma árabe, también es una selección adecuada a la hora de querer interpretar aquellas estructuras caracterizadas por su percepción inespecífica debido a la ausencia del sujeto. De esta manera, tanto el sujeto de la impersonales con se, en español, como el de las oraciones en voz no-agentiva, en árabe, pueden interpretarse de forma similar a 'todo el mundo', 'cualquiera' o 'alguien'.

d. En un mecanismo creado por el idioma para evitar las redundancias no necesarias, el español emplea el pronombre reflexivo se para indicar que la acción del verbo recae sobre el propio sujeto o sobre una parte del cuerpo o la ropa de ese sujeto. En contraste, la lengua árabe, al carecer de pronombres reflexivos, basándose en el paradigma morfológico árabe, cuenta con un o unos morfemas derivacionales ligados al lexema del verbo para referirse a que el efecto del verbo se refleja sobre el propio sujeto. En cuanto a las reflexivas indirectas, los pronombres personales que funcionan como posesivos cuando

(Uses of (SE) Contrastive Analysis...) Dr. Mohamed Shebl 


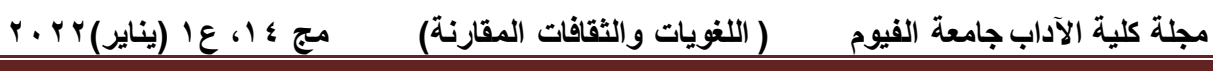

se sufijan a un nombre, son los que se encargan de desempeñar ese papel.

e. El pronombre recíproco se indica que cada individuo de un sujeto múltiple ejecuta la acción verbal hacia el otro o los otros. Por su parte, el idioma árabe, para expresar dicha reciprocidad, fundamentándose también en el modelo morfológico árabe, presenta varias fórmulas morfológicas dotadas de expresar tal valor semántico.

f. En torno al dativo ético se, que funciona como intensificador del proceso verbal, su aparición en estructuras transitivas o intransitivas es marginal, por lo que puede suprimirse sin que cambie el significado en esencia. Por otra parte, el idioma árabe, para expresar dicho valor semántico tiene otros mecanismos, entre los cuales se distingue el estilo de la afirmación semántica.

(Uses of (SE) Contrastive Analysis...) Dr. Mohamed Shebl 


\section{Referencias bibliográficas}

A. de Molina Redondo, J. (1990). Usos de «SE»: cuestiones sintácticas y léxicas. Madrid: SGEL.

Algalayeny, Mustafa. (2016). Gāmi al-durws al-arabiyyah (Coleccionista de las clases de árabe). T. I. Beirut: Dar AlKotob Al-Ilmiyah.

Alsirafy, Abu Saed. (2017). Šaḥ kitāb Sibawayh (Explicación del Libro de Sibawayh). T. III. Beirut: Dar Al-Kotob AlIlmiyah.

Awad Al-seyori, Abdul-Hamid (ed.). (2014/2015). Al-qawā'id al-'asāsiyyah lil-naḥw wal-ṣarf (Normas básicas de sintaxis y morfología). Egipto: Ministerio de Educación y Enseñanza. Cascón Martín, Eugenio. (1996). Sintaxis: Teoría y práctica del análisis oracional. Madrid: Edinumen.

Corriente, Federico (2006). Gramática Árabe. Barcelona: Herder.

Diez de la Cortina Montemayor, Susana. (2012). Fichas gramaticales de la lengua española (14. Pronombres personales III: función de complemento). Madrid: Manuscritos (edición electrónica).

Fayaad, Sulaiman. (1995). Al- nạ̣w al-așriyy (Sintaxis contemporánea). El-Cairo: Centro de Al-Ahram.

(Uses of (SE) Contrastive Analysis...) Dr. Mohamed Shebl 
Gawad Altereehy, M. (2014). Islūb șiyag afāl al-mušārakah wa mașādirihā fī al-'adā' al-nahawiyy (Método de las formas de verbos de reciprocidad y su infinitivo en el funcionamiento sintáctico). Revista de la Facultad de Letras-Universidad de Baghdad, s. n., pp. 1-34. Recuperado de https://ar.islamway.net/book/11597/

Gili Gaya, Samuel. 1991. Curso superior de sintaxis española. Barcelona: Indugraf.

Gómez Torrego, Leonardo. (2005). Valores Gramaticales de "SE". Madrid: Arco Libro.

González Hermoso, A., Cuenot, J. R. y Sánchez Alfaro, M. (2006). Gramática de la lengua española. Madrid: Edelsa. Gradat, Osama Kamel. (2004). Al-'ab'àd al-manawiyya fi alwazā'if al-nahawiyyah (Dimensiones semánticas de las funciónes gramaticales). Argelia: Dar-alfuruan.

Gutiérrez Araus, María Luz et.al. (2010). Curso básico de lengua española. Madrid: Centro de Estudios Ramón Areces. Ibn 'Aqīl, 'Abd Allāh. (1980). Šarḥ Ibn 'Aqīl (Explicación de Ibn 'Aqīl), T. II. Egipto: Dar Al-Turath.

Moreno-Fernández, Francisco, Penadés-Martínez, Inmaculada y Ureña-Tormo, Clara. (2020). Gramática fundamental del español. Oxon y Nueva York: Routledge.

(Uses of (SE) Contrastive Analysis...) Dr. Mohamed Shebl 


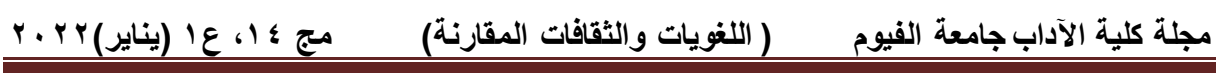

Mustafa Aldamj, K. (2016). Al-nojba al-șirf: men aḥām ilm alșarf (La mera elección: de los procedimientos de la morfología). Beirut: Ed. Dar Al-Kotob Al-Ilmiyah.

Navarro Lacoba, Rocío. 2014. Las oraciones pasivas: teoría y ejemplos. Madrid: Rocío Navarro Lacoba

Porto Dapena, J. Alvaro. (1986). Los pronombres. Madrid:

Selecciones Gráficas.

Sánchez López, Cristina (ed.). (2002). Las construcciones con 'se'. Madrid: Visor Libros.

Sarmiento, Ramón y Sánchez Aquilino. (1989). Gramática básica del español: norma y uso. Madrid: SGEL.

(Uses of (SE) Contrastive Analysis...) Dr. Mohamed Shebl 


\section{استخدامات (SE): تحليل تقابلي بين الإسبانية وإلعربية}

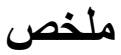

هي كثثرة تلك الدراسات التي تتناول التحليل المقارن وأهميته ومساهمته في مجال التعلّم والتعليم للغات الأجنبية، باعتباره وسيلة فعالة لتطوير المواد الدراسية الملائمة، ولتدريب مدرسي اللغة الإسبانية كلغة أجنبية ليكونوا على دراية بطبيعة وعقلية الطلاب الذين يسعون لاكتساب تلك اللغة. فالتحليل المقارن يسمح للمعلمين بالتتبؤ بالمشاكل التي قد يواجهها طلابهم، وتحديد درجة الصعوبة التي تمثلها لهم، وبالتالي تحديد أفضل الطرق لحلها. لهذا السبب، وانطلاقًا من قناعتنا التامة بجدوى ذلك المنهج التعليمي، فقد اعتمدناه لإجراء مقابلة بين الإسبانية والعربية من حيث الاستخدامات اللغوية المتعددة للمكون اللغوي (se) في اللغة الإسبانية والوحدات اللغوية المقابلة في اللغة العربية. حيث تكمن صعوبة ادراك وفهم الدارس ذو الأصل العربي لذلك المكون النحوي في الإسبانية لكثرة دلالاته، وتعدد استخداماته، ولعدم وجود كلمة لها نفس الطبيعة في لغته الأم يمكن استخدامها بنفس الكيفية، فهو ضمير شخصي، وأداة بناء للمجهول، وضمير منعكس، وضمير دال على المشاركة، وأداة تعميم لفاعل الجملة، وضمير تأكيد لمعنى الفعل في علاقته بالفاعل.

الكلمات المفتاحية:تحليل تقابلي، الفاعل، نائب الفاعل، المبني للمجهول، بنية الكلمة.

(Uses of (SE) Contrastive Analysis...) Dr. Mohamed Shebl 